\title{
GROWTH PROPERTY FOR THE MINIMAL SURFACE EQUATION IN UNBOUNDED DOMAINS
}

\author{
JENN-FANG HWANG
}

(Communicated by Peter Li)

\begin{abstract}
Here we prove that if $u$ satisfies the minimal surface equation in an unbounded domain $\Omega$ which is properly contained in a half plane, then the growth rate of $u$ is of the same order as the shape of $\Omega$ and $\left.u\right|_{\partial \Omega}$.
\end{abstract}

\section{INTRODUCTION}

The purpose of this paper is to improve a Phragmèn-Lindelöf Theorem for the minimal surface equation in $\mathbb{R}^{2}$. Hwang has proved that if $u$ satisfies the minimal surface equation in an unbounded domain $\Omega$, which is properly contained in a half plane, the growth property of $u$ depends on $\Omega$ and $\left.u\right|_{\partial \Omega}$ only, without requiring any restriction for $u$ [3]. In this respect, the PhragmènLindelöf Theorem for the minimal surface equation is better than that of the Laplace equation. We remark that if $u$ satisfies the Laplace equation in an unbounded domain $\Omega$, the growth property of $u$ cannot be determined completely by the shape of $\Omega$ and $\left.u\right|_{\partial \Omega}$ alone [7].

But the estimate in [3] is not good enough. For example, let $\Omega=\{-\cosh y<$ $x<\cosh y \mid y>0\}$ and $\operatorname{div} T u=0$ in $\Omega,\left.u\right|_{\partial \Omega}=\left.\sqrt{(\cosh y)^{2}-x^{2}}\right|_{\partial \Omega}$. Then, by Example 3.4 of [3], we know that $u=O\left(y e^{y}\right)$ as $y \rightarrow \infty$, but the growth rate of the solution $\sqrt{(\cosh y)^{2}-x^{2}}$ (catenoid) is $O\left(e^{y}\right)$.

The purpose of this paper is to improve the estimate of [3]. We will prove that the growth rate of $u$ is of the same order as the shape of $\Omega$ and $\left.u\right|_{\partial \Omega}$ (Theorems 2.12 and 2.13). In fact, let $\Omega=\{-\cosh y<x<\cosh y\}$. We will prove that a catenoid is the maximum solution among the solutions with vanishing boundary value (Corollary 2.3 ).

\section{PhragmÉn-LindelöF TheOREMS FOR $\mathbb{R}^{2}$}

Throughout the paper, $\Omega$ will be a connected domain (bounded or unbounded) in $\mathbb{R}^{2}$ and, for any function $u \in C^{2}(\Omega), T u$ will denote the vector $D u / \sqrt{1+|D u|^{2}}$, where $D u$ is the gradient vector of $u$ and the minimal surface

Received by the editors September 22, 1992.

1991 Mathematics Subject Classification. Primary 35B05; Secondary 35J10, 49Q05, 53A10. 
operator $\mathfrak{M}$ is given by

$$
\mathfrak{M} u=\left(1+|D u|^{2}\right) \Delta u-D_{i} u D_{j} u D_{i j} u=\left(1+|D u|^{2}\right)^{3 / 2} \operatorname{div} T u,
$$

where $\Delta u$ is the Laplacian of $u$.

We will use functions of the form

$$
F(x, y)=(G(x, y))^{1 / 2} g(y)+h(y)
$$

as comparison functions, and we compute $\mathfrak{M F}$ in the following lemma.

\section{Lemma 2.1. Let}

$$
F(x, y)=(G(x, y))^{1 / 2} g(y)+h(y),
$$

where $F, G: \mathbb{R}^{2} \rightarrow \mathbb{R}^{1}, g, h: \mathbb{R}^{1} \rightarrow \mathbb{R}^{1}, F, G, g, h \in C^{2}$, and $G>0$. Then $G^{3 / 2} \mathfrak{M F}=I+I I+I I I$, where

$$
\begin{aligned}
I= & g^{3}\left(\frac{1}{4} G_{x}^{2}\left(\frac{1}{2} G_{y y}+G \frac{g^{\prime \prime}}{g}-3 G\left(\frac{g^{\prime}}{g}\right)^{2}\right)\right. \\
& \left.\quad+\frac{1}{2} G_{x x}\left(\frac{1}{4} G y^{2}+G_{y} G \frac{g^{\prime}}{g}+G^{2}\left(\frac{g^{\prime}}{g}\right)^{2}\right)-\frac{1}{2} G_{x} G_{x y}\left(\frac{1}{2} G_{y}+G \frac{g^{\prime}}{g}\right)\right), \\
I I= & g\left(-\frac{1}{4} G_{y}^{2}+\frac{1}{2} G G_{y y}+G G_{y} \frac{g^{\prime}}{g}+G^{2} \frac{g^{\prime \prime}}{g}-\frac{1}{4} G_{x}^{2}+\frac{1}{2} G_{x x} G\right), \\
I I I= & G^{1 / 2} h^{\prime \prime}\left(G+\frac{1}{4} G_{x}^{2} g^{2}\right) \\
& +G^{1 / 2} h^{\prime}\left(-\frac{1}{2} G_{x} G_{x y} g^{2}-G_{x}^{2} g g^{\prime}+\frac{1}{2} G_{y} G_{x x} g^{2}+G G_{x x} g g^{\prime}\right) \\
& +h^{\prime 2}\left(-\frac{1}{4} G_{x}^{2} g+\frac{1}{2} G G_{x x} g\right) .
\end{aligned}
$$

Proof. By simple computation we have

$$
\begin{gathered}
\left(1+F_{x}^{2}\right) F_{y y}=\left(1+\frac{1}{4} G^{-1} G_{x}^{2} g^{2}\right) \\
\times\left(-\frac{1}{4} G^{-3 / 2} G_{y}^{2} g+\frac{1}{2} G^{-1 / 2} G_{y y} g+G^{-1 / 2} G_{y} g^{\prime}+G^{1 / 2} g^{\prime \prime}+h^{\prime \prime}\right) \\
-2 F_{x} F_{y} F_{x y}=-2\left(\frac{1}{2} G^{-1 / 2} G_{x} g\right)\left(\frac{1}{2} G^{-1 / 2} G_{y} g+G^{1 / 2} g^{\prime}+h^{\prime}\right) \\
\times\left(-\frac{1}{4} G^{-3 / 2} G_{x} G_{y} g+\frac{1}{2} G^{-1 / 2} G_{x y} g+\frac{1}{2} G^{-1 / 2} G_{x} g^{\prime}\right) \\
\left(1+F_{y}^{2}\right) F_{x x}=\left(1+\left(\frac{1}{2} G^{-1 / 2} G_{y} g+G^{1 / 2} g^{\prime}+h^{\prime}\right)^{2}\right) \\
\times\left(-\frac{1}{4} G^{-3 / 2} G_{x}^{2} g+\frac{1}{2} G^{-1 / 2} G_{x x} g\right)
\end{gathered}
$$


Hence

$$
\begin{aligned}
G^{5 / 2} \mathfrak{M} F= & \left(G+\frac{1}{4} G_{x}^{2} g^{2}\right)\left(-\frac{1}{4} G_{y}^{2} g+\frac{1}{2} G G_{y y} g+G G_{y} g^{\prime}+G^{2} g^{\prime \prime}+G^{3 / 2} h^{\prime \prime}\right) \\
& -2\left(\frac{1}{2} G_{x} g\right)\left(\frac{1}{2} G_{y} g+G g^{\prime}+G^{1 / 2} h^{\prime}\right)\left(-\frac{1}{4} G_{x} G_{y} g+\frac{1}{2} G G_{x y} g+\frac{1}{2} G G_{x} g^{\prime}\right) \\
& +\left(G+\left(\frac{1}{2} G_{y} g+G g^{\prime}+h^{\prime} G^{1 / 2}\right)^{2}\right)\left(-\frac{1}{4} G_{x}^{2} g+\frac{1}{2} G G_{x x} g\right) \\
= & \frac{1}{4} G_{x}^{2} g^{2}\left(-\frac{1}{4} G_{y}^{2} g+\frac{1}{2} G G_{y y} g+G G_{y} g^{\prime}+G^{2} g^{\prime \prime}\right) \\
& -2\left(\frac{1}{2} G_{x} g\right)\left(\frac{1}{2} G_{y} g+G g^{\prime}\right)\left(-\frac{1}{4} G_{x} G_{y} g+\frac{1}{2} G G_{x y} g+\frac{1}{2} G G_{x} g^{\prime}\right) \\
& +\left(\frac{1}{2} G_{y} g+G g^{\prime}\right)^{2}\left(-\frac{1}{4} G_{x}^{2} g+\frac{1}{2} G G_{x x} g\right) \\
& +G\left(-\frac{1}{4} G_{y}^{2} g+\frac{1}{2} G G_{y y} g+G G_{y} g^{\prime}+G^{2} g^{\prime \prime}-\frac{1}{4} G_{x}^{2} g+\frac{1}{2} G_{x x} g G\right) \\
& +G^{3 / 2} h^{\prime \prime}\left(G+\frac{1}{4} G_{x}^{2} g^{2}\right) \\
& +G^{1 / 2} h^{\prime}\left(-G_{x} g\right)\left(-\frac{1}{4} G_{x} G_{y} g+\frac{1}{2} G G_{x y} g+\frac{1}{2} G G_{x} g^{\prime}\right) \\
& +2 h^{\prime} G^{1 / 2}\left(\frac{1}{2} G_{y} g+G g^{\prime}\right)\left(-\frac{1}{4} G_{x}^{2} g+\frac{1}{2} G G_{x x} g\right) \\
& +h^{\prime 2} G\left(-\frac{1}{4} G_{x}^{2} g+\frac{1}{2} G G_{x x} g\right) \\
= & g^{3}\left(\frac{1}{4} G_{x}^{2}\left(\frac{1}{2} G G_{y y}+G^{2} \frac{g^{\prime \prime}}{g}-3 G^{2}\left(\frac{g^{\prime}}{g}\right)^{2}\right)\right. \\
& \left.+\frac{1}{2} G G_{x x}\left(\frac{1}{2} G_{y}+G_{\frac{g^{\prime}}{g}}\right)^{2}-\frac{1}{2} G G_{x} G_{x y}\left(\frac{1}{2} G_{y}+G_{\frac{g^{\prime}}{g}}\right)\right) \\
& +G g\left(-\frac{1}{4} G_{y}^{2}+\frac{1}{2} G G_{y y}+G G_{y} \frac{g^{\prime}}{g}+G^{2} \frac{g^{\prime \prime}}{g}-\frac{1}{4} G_{x}^{2}+\frac{1}{2} G_{x x} G\right) \\
& +G\left(G^{1 / 2} h^{\prime \prime}\left(G+\frac{1}{4} G_{x}^{2} g^{2}\right)\right. \\
& +G^{1 / 2} h^{\prime}\left(-\frac{1}{2} G_{x} G_{x y} g^{2}-G_{x}^{2} g g^{\prime}+\frac{1}{2} G_{y} G_{x x} g^{2}+G G_{x x} g g^{\prime}\right) \\
& \left.+h^{2}\left(-\frac{1}{4} G_{x}^{2} g+\frac{1}{2} G G_{x x} g\right)\right) \\
= & G(I+I I+I I I) .
\end{aligned}
$$

The lemma follows.

Now we treat the Phragmèn-Lindelöf Theorem for comparison functions $H(x, y)$ with faster growth. Since, in a half plane, the bound of the solutions with vanishing boundary value does not even exist, the domain must be properly contained in a half plane.

Lemma 2.2. Let $H(x, y)=a \sqrt{f^{2}(y)-x^{2}}$ and $\Omega \subset\left\{-f_{1}(y)<x<f_{1}(y), y>\right.$ $0\}$, where $f, f_{1}:[0, \infty) \rightarrow[0, \infty), f \in C^{2}, f_{1} \in C^{0}, f \geq f_{1}>0$ for $y>$ $0, f^{\prime} \equiv \frac{d f}{d y}>0$, and $a$ is a positive constant. Let $u \in C^{2}(\Omega) \cap C^{0}(\bar{\Omega})$, and suppose that

(i) $\operatorname{div} T H-\operatorname{div} T u \leq 0$ in $\Omega$,

(ii) $\left.(u-H)\right|_{\partial \Omega} \leq 0$,

(iii) $\liminf _{y \rightarrow \infty} f_{1}(y) / f^{\prime 2}=0$.

Then $u \leq H$ in $\Omega$.

Remark. It is easy to see that if $\lim _{y \rightarrow \infty} f(y) / f^{\prime 2}=0$, then the rate of growth of $f$ must be faster than $y^{2}$ as $y \rightarrow \infty$.

Proof of Lemma 2.2. If $\{(x, y) \in \Omega \mid u(x, y)-H(x, y)>0\}$ is nonempty, there exists $\varepsilon>0$ such that $\Omega^{\prime}=\{(x, y) \in \Omega \mid u(x, y)-H(x, y)>\varepsilon\}$ is non-empty and $\partial \Omega^{\prime} \cap \Omega$ is smooth (Sard's Theorem). Since $\left.(u-H)\right|_{\partial \Omega} \leq 0$, we have $\partial \Omega^{\prime} \subset \Omega$ and $\partial \Omega^{\prime}=\{(x, y) \in \Omega \mid u-H=\varepsilon\}$. 
For every $y_{0}>0$, let $\Omega_{y_{0}}=\Omega^{\prime} \cap\left\{y<y_{0}\right\}$ and $\Gamma_{y_{0}}=\partial \Omega_{y_{0}} \cap\left\{y=y_{0}\right\}$. By the divergence theorem, we have

$$
\begin{aligned}
\int_{\partial \Omega_{y_{0}}} & \tan ^{-1}(u-H-\varepsilon)(T u-T H) \cdot \nu d \sigma \\
= & \iint_{\Omega_{y_{0}}} \frac{(D u-D H)}{1+(u-H-\varepsilon)^{2}}(T u-T H) d x \\
& +\iint_{\Omega_{y_{0}}}(u-H-\varepsilon)(\operatorname{div} T u-\operatorname{div} T H) d x,
\end{aligned}
$$

where $\nu$ is the unit outer normal of $\partial \Omega_{y_{0}}$. Noticing that $\partial \Omega_{y_{0}} \backslash \Gamma_{y_{0}} \subset \partial \Omega^{\prime}$, we have $u-H=\varepsilon$ on $\partial \Omega_{y_{0}} \mid \Gamma_{y_{0}}$. Since $\tan ^{-1}(u-H-\varepsilon)(\operatorname{div} T u-\operatorname{div} T H) \geq 0$ in $\Omega_{y_{0}}$ and $T u \cdot \nu \leq 1$,

$$
\begin{aligned}
& \iint_{\Omega_{y_{0}}} \frac{(D u-D H)}{1+(u-H-\varepsilon)^{2}}(T u-T H) d x \\
& \quad \leq \int_{\Gamma_{y_{0}}} \tan ^{-1}(u-H-\varepsilon)(T u-T H) \cdot \nu d \sigma \\
& \quad \leq \int_{\Gamma_{y_{0}}} \tan ^{-1}(u-H-\varepsilon)(1-T H \cdot \nu) d \sigma .
\end{aligned}
$$

Since $\nu=\langle 0,1\rangle$ on $\Gamma_{y_{0}}$,

$$
\begin{gathered}
H=a \sqrt{f^{2}-x^{2}}, \quad H_{y}=a \frac{f f^{\prime}}{\sqrt{f^{2}-x^{2}}}, \quad H_{x}=-\frac{a x}{\sqrt{f^{2}-x^{2}}}, \\
\left.T H \cdot \nu\right|_{\Gamma_{y_{0}}}=\frac{H_{y}}{\sqrt{1+H_{x}^{2}+H_{y}^{2}}}=\frac{a f f^{\prime} / \sqrt{f^{2}-x^{2}}}{\sqrt{1+a^{2} x^{2} /\left(f^{2}-x^{2}\right)+a^{2} f^{2} f^{\prime 2} /\left(f^{2}-x^{2}\right)}} \\
=\frac{a f f^{\prime}}{\sqrt{f^{2}-x^{2}+a^{2} x^{2}+a^{2} f^{2} f^{\prime 2}}}, \\
\left.(1-T H \cdot \nu)\right|_{\Gamma_{y_{0}}} \\
=\frac{\sqrt{f^{2}-x^{2}+a^{2} x^{2}+a^{2} f^{2} f^{\prime 2}}-a f f^{\prime}}{\sqrt{f^{2}-x^{2}+a^{2} x^{2}+a^{2} f^{2} f^{\prime 2}}} \\
\quad \cdot \frac{\sqrt{f^{2}-x^{2}+a^{2} x^{2}+a^{2} f^{2} f^{\prime 2}}+a f f^{\prime}}{\sqrt{f^{2}-x^{2}+a^{2} x^{2}+a^{2} f^{2} f^{\prime 2}}+a f f^{\prime}} \\
=\frac{f^{2}-x^{2}+a^{2} x^{2}}{\sqrt{f^{2}-x^{2}+a^{2} x^{2}+a^{2} f^{2} f^{\prime 2}}\left(\sqrt{f^{2}-x^{2}+a^{2} x^{2}+a^{2} f^{2} f^{\prime 2}}+a f f^{\prime}\right)} \\
\leq \leq \frac{\left(1+a^{2}\right) f^{2}}{a^{2} f^{2} f^{\prime 2}}=\frac{1+a^{2}}{a^{2} f^{\prime 2}} .
\end{gathered}
$$


Now

$$
\begin{aligned}
& \iint_{\Omega_{y_{0}}} \frac{(D u-D H)}{1+(u-H-\varepsilon)^{2}}(T u-T H) d x \\
& \quad \leq \int_{\Gamma_{y_{0}}} \tan ^{-1}(u-H-\varepsilon)(1-T H \cdot \nu) d \sigma \\
& \quad \leq \frac{\pi}{2} \int_{\Gamma_{y_{0}}} \frac{1+a^{2}}{a^{2} f^{\prime 2}} d \sigma \leq \pi \cdot \frac{\left(1+a^{2}\right) f_{1}}{a^{2} f^{\prime 2}}\left(y_{0}\right) .
\end{aligned}
$$

Let $y_{0} \rightarrow \infty$. We have

$$
0 \leq \iint_{\Omega^{\prime}} \frac{(D u-D H)}{1+(u-H-\varepsilon)^{2}}(T u-T H) d x \leq 0 .
$$

Since $(D u-D H) \cdot(T u-T H) \geq 0$ and the equality holds when $D u=D H$, we have $D u-D H \equiv 0$ in $\Omega^{\prime}$. Then $u \equiv H+\varepsilon$ in $\Omega^{\prime}$, and, by definition, $\Omega^{\prime}$ must be empty. This is impossible, and we conclude that $u(x) \leq H(x)$ for all $x$ in $\Omega$.

Remark. The following well-known fact is used to prove Lemma 2.2: $D u / \sqrt{1+|D u|^{2}}$ has norm less than 1 . It is a very important idea for the capillary surface equation (cf. [1, Theorem 5.1]).

Now we obtain the result: catenoid is the maximum solution among those surfaces on $\Omega=\{-\cosh y<x<\cosh y\}$ satisfying the minimal surface equation and with vanishing boundary value.

Corollary 2.3. Let $\Omega=\{-\cosh y<x<\cosh y\}$, and let $u \in C^{2}(\Omega) \cap C^{0}(\Omega)$. Suppose that

(i) $\operatorname{div} T u \geq 0$ in $\Omega$,

(ii) $\left.u\right|_{\partial \Omega} \leq 0$.

Then $u \leq \sqrt{(\cosh y)^{2}-x^{2}}$.

Proof. The corollary can be proved by the fact that

$$
\operatorname{div} T u-\operatorname{div} T \sqrt{(\cosh y)^{2}-x^{2}} \geq 0, \quad \lim _{y \rightarrow \pm \infty} \frac{\cosh y}{\left[(\cosh y)^{\prime}\right]^{2}}=0
$$

and a similar argument as in the proof of Lemma 2.2.

By Lemma 2.2, it is sufficient to show that $\operatorname{div} T\left(a \sqrt{f^{2}-x^{2}}\right) \leq 0$ holds when $\liminf _{y \rightarrow \infty}\left(f_{1} / f^{\prime 2}\right)=0$. Let $f \in C^{2}, f \geq 0$, and $f^{\prime}>0$. Define $p(f)=\left(1 /(\log f)^{\prime}\right)^{\prime}=\left(f^{\prime 2}-f f^{\prime \prime}\right) / f^{\prime 2}=1-f f^{\prime \prime} / f^{\prime 2}$; some properties of $p(f)$ will be discussed later. By setting $F=a \sqrt{f^{2}-x^{2}}, G=f^{2}-x^{2}$, and $g=a$ in Lemma 2.1 where $a$ is a positive constant, we get

$$
\begin{aligned}
G^{3 / 2} \mathfrak{M} F=a^{3}\left(x^{2} f^{\prime 2}+x^{2} f f^{\prime \prime}-f^{2}{f^{\prime}}^{2}\right)+a\left(f f^{\prime \prime}\left(f^{2}-x^{2}\right)-x^{2}{f^{\prime}}^{2}-f^{2}\right) \\
\quad=f^{\prime 2} a^{3}\left(-f^{2}+(2-p(f)) x^{2}\right)+f^{\prime 2} a\left(f^{2}(1-p(f))-(2-p(f)) x^{2}\right)-a f^{2} \\
\quad=-\left(a-a^{3}\right)(2-p(f)) x^{2}{f^{\prime}}^{2}+f^{2} f^{\prime 2} a\left((1-p(f))-a^{2}\right)-a f^{2} .
\end{aligned}
$$

Lemma 2.4. Let $f, p(f), F, G$, and $a$ be defined as above, and let $\Omega \subset$ $\left\{(x, y) \mid-f_{1}(y)<x<f_{1}(y), y>0\right\}$, where $f_{1}>0$ is given. Suppose $p(f) \geq p_{0}$, where $p_{0}$ is a constant. Then

(i) if $1 \geq p_{0} \geq 0, a=\sqrt{1-p_{0}}$, and $f \geq f_{1}$, or 
(ii) if $p_{0}<0, a>\sqrt{1-p_{0}}, a$ is a positive constant, and $f^{2} \geq$ $\left(a^{2}-1\right)\left(2-p_{0}\right) f_{1}^{2} /\left(a^{2}-\left(1-p_{0}\right)\right)$,

we have $G^{3 / 2} \mathfrak{M} F \leq 0$ in $\Omega$.

Proof. (i) If $2 \geq p(f) \geq p_{0}$, it is easy to see that

$$
G^{3 / 2} \mathfrak{M} F \leq 0
$$

If $p(f) \geq 2$, we have

$$
G^{3 / 2} \mathfrak{M} F \leq(p(f)-2) \sqrt{1-p_{0}} p_{0} x^{2} f^{\prime 2}+f^{2} f^{\prime 2} \sqrt{1-p_{0}}\left(-p(f)+p_{0}\right) \leq 0 .
$$

(ii) By assumption, it is easy to have the following:

$$
\begin{aligned}
G^{3 / 2} \mathfrak{M} F= & f^{\prime 2} a^{3}\left(-f^{2}+(2-p(f)) x^{2}\right) \\
& +f^{\prime 2} a\left(f^{2}(1-p(f))-(2-p(f)) x^{2}\right)-a f^{2} \\
= & a f^{\prime 2}\left(\left(a^{2}-1\right)(2-p(f)) x^{2}-f^{2}\left(a^{2}-(1-p(f))\right)\right)-a f^{2} \leq 0 .
\end{aligned}
$$

Hence it is easy to derive the following theorem.

Theorem 2.5. Let $f, f_{1}, p(f)$, and $\Omega$ be defined as in Lemma 2.4. Let $\liminf _{y \rightarrow \infty}\left(f_{1} / f^{\prime 2}\right)=0, u \in C^{2}(\Omega) \cap C^{0}(\Omega)$, and $\operatorname{div} T u \geq 0$ in $\Omega$. Then

(i) if $p(f) \geq p_{0} \geq 0$ where $p_{0}$ is a constant, $f \geq f_{1}, 1 \geq p_{0} \geq 0$, and $\left.u\right|_{\partial \Omega} \leq\left.\sqrt{1-p_{0}} \sqrt{f^{2}-x^{2}}\right|_{\partial \Omega}$, then we have $u \leq \sqrt{1-p_{0}} \sqrt{f^{2}-x^{2}}$ in $\Omega$,

(ii) if $p(f) \geq p_{0}$, where $p_{0}$ is a negative constant, and

$$
f^{2} \geq \frac{\left(a^{2}-1\right)\left(2-p_{0}\right)}{a^{2}-\left(1-p_{0}\right)} f_{1}^{2},\left.\quad u\right|_{\partial \Omega} \leq\left. a \sqrt{f^{2}-x^{2}}\right|_{\partial \Omega},
$$

where $a$ is a positive constant satisfying $a^{2}-1+p_{0}>0$, then we have $u \leq$ $a \sqrt{f^{2}-x^{2}}$ in $\Omega$.

We will show later that the above theorem still remains valid without the condition $\liminf _{y \rightarrow \infty}\left(f_{1} / f^{\prime 2}\right)=0$. We first investigate the properties of $p(f)$. Let $f \in C^{2}, f \geq 0, f^{\prime}>0, a$ be a positive constant, and $\alpha \neq 0$ be a constant. Since $p\left(a f^{\alpha}\right)=\left(1 /\left(\log \left(a f^{\alpha}\right)\right)^{\prime}\right)^{\prime}=\frac{1}{\alpha}\left(1 /(\log f)^{\prime}\right)^{\prime}$, we have $p\left(a f^{\alpha}\right)=\frac{1}{\alpha} p(f)$. Then we prove the following lemma.

Lemma 2.6. Let $f, f_{1} \in C^{2}$, and $f, f^{\prime}, f_{1}, f_{1}^{\prime}>0$. Then

(i) if $p(f), p\left(f_{1}\right) \geq p_{0}>0$, where $0<p_{0} \leq 1$, is a constant, then $p\left(f f_{1}\right) \geq$ $p_{0} / 2>0$;

(ii) if $p(f) \geq 0$, then $p\left(e^{a y} f\right) \geq 0$ for any positive constant $a$;

(iii) if $p(f) \geq p_{0}$, where $p_{0}$ is a negative constant, then $p\left(e^{a y} f\right) \geq p_{0}$ for any positive constant $a$.

Proof. (i) Let $h=(\log f)^{\prime}=f^{\prime} / f>0$ and let $h_{1}=\left(\log f_{1}\right)^{\prime}=f_{1}^{\prime} / f_{1}>0$. Since $p(f)=(1 / h)^{\prime}=-h^{\prime} / h^{2} \geq p_{0}>0$ and $p\left(f_{1}\right)=\left(1 / h_{1}\right)^{\prime}=-h_{1}^{\prime} / h_{1}^{2} \geq p_{0}>0$, we have $p\left(f f_{1}\right)=\left(1 /\left(h+h_{1}\right)\right)^{\prime}=-\left(h^{\prime}+h_{1}^{\prime}\right) /\left(h+h_{1}\right)^{2} \geq-\left(h^{\prime}+h_{1}^{\prime}\right) / 2\left(h^{2}+h_{1}^{2}\right) \geq$ $p_{0} / 2$.

(ii) Since $p(f)=-h^{\prime} / h^{2} \geq 0$, we have $p\left(e^{a y} f\right)=\left(1 /\left(\left(\log e^{a y}\right)^{\prime}+(\log f)^{\prime}\right)\right)^{\prime}$ $=(1 /(a+h))^{\prime}=-h^{\prime} /(a+h)^{2} \geq 0$.

(iii) Since $p(f)=-h^{\prime} / h^{2}$ and $p\left(e^{a y} f\right)=-h^{\prime} /(a+h)^{2}$ have the same sign and $\left|p\left(e^{a y} f\right)\right| \leq|p(f)|$, the result follows immediately. 
Remark 2.7. (i) If $f=y^{m}$, where $m$ is a positive constant, then $p(f)=\frac{1}{m}$.

(ii) $p\left(e^{y}\right)=0$.

(iii) If we set $f=e^{y^{\alpha}}$, where $\alpha>1$ is a constant, then $p(f)=\left(1 /\left(y^{\alpha}\right)^{\prime}\right)^{\prime}=$ $\left(y^{1-\alpha} / \alpha\right)^{\prime}=(1-\alpha) y^{-\alpha} / \alpha$. This implies that $p(f) \rightarrow 0^{-}$as $y \rightarrow \infty$, so, for sufficiently large $y$, we have $p(f)>-\varepsilon$ for some small positive number $\varepsilon$.

Similarly, if $f$ increases faster than the exponential function, then we can assume $p(f) \geq-\varepsilon$ for some small positive constant $\varepsilon$ essentially.

Lemma 2.8. Let $f \in C^{1}, f, f^{\prime}>0$, and $\lim \sup _{y \rightarrow \infty}\left(f(y) / y^{2}\right)=+\infty$. Then $\liminf _{y \rightarrow \infty}\left(f / f^{\prime 2}\right)=0$.

Proof. Suppose not; then there exist positive constants $y_{0}$ and $C$ such that, for every $y>y_{0}$, we have $f(y) / f^{\prime 2}(y) \geq C$, so $f=O\left(y^{2}\right)$. Contradiction arises and the lemma follows.

We are now ready to remove the condition $\liminf _{y \rightarrow \infty}\left(f / f^{\prime 2}\right)=0$ in Theorem 2.5.

We will start with a theorem.

Theorem 2.9. Let $f \in C^{0}[0, \infty) \cap C^{2}(0, \infty), f \geq 0, f^{\prime}>0, \Omega \subset\{(x, y) \mid$ $-f(y)<x<f(y), y>0\}, u \in C^{2}(\Omega) \cap C^{0}(\bar{\Omega})$, $\operatorname{div} T u \geq 0$, and $p(f) \geq p_{0} \geq$ 0 , where $p_{0}$ is a constant. Then

(i) if $p_{0}=0$ and $\left.\left(u-\sqrt{f^{2}-x^{2}}\right)\right|_{\partial \Omega} \leq 0$, we have $u \leq \sqrt{f^{2}-x^{2}}$ in $\Omega$,

(ii) if $1 \geq p_{0}>0$ and $\left.\left(u-\sqrt{1-q_{0}} \sqrt{f^{2}-x^{2}}\right)\right|_{\partial \Omega} \leq 0$, where $q_{0}=$ $\min \left(1 / 8, p_{0} / 2\right)$, we have $u \leq \sqrt{1-q_{0}} \sqrt{f^{2}-x^{2}}$ in $\Omega$.

Proof. (i) Let $h=e^{a y} f$, where $a$ is a positive constant. Then by Lemma 2.6 $p(h) \geq 0$. So $\mathfrak{M}\left(\sqrt{h^{2}-x^{2}}\right) \leq 0$ in $\Omega$. Since $f^{\prime}>0, \limsup _{y \rightarrow \infty}\left(h / y^{2}\right)=$ $+\infty$. By Lemma $2.8, \liminf _{y \rightarrow \infty}\left(h / h^{\prime 2}\right)=0$. Since $\left.u\right|_{\partial \Omega} \leq\left.\sqrt{f^{2}-x^{2}}\right|_{\partial \Omega} \leq$ $\left.\sqrt{h^{2}-x^{2}}\right|_{\partial \Omega}$, by Theorem 2.5, $u \leq \sqrt{h^{2}-x^{2}}=\sqrt{\left(e^{a y} f\right)^{2}-x^{2}}$ in $\Omega$. Setting $a \rightarrow 0$, the result follows.

(ii) Let $h=(1+b y)^{4} f$, where $b$ is a positive constant. Since $p\left((1+b y)^{4}\right)=\frac{1}{4}$, by Lemma $2.6, p(h) \geq \min \left(1 / 4, p_{0}\right) / 2=q_{0}>0$. By Lemma 2.4, $\mathfrak{M}\left(\sqrt{1-q_{0}} \sqrt{\left.h^{2}-x^{2}\right)} \leq 0\right.$ in $\Omega$. Since $f^{\prime}>0, \limsup _{y \rightarrow \infty}\left(h / y^{2}\right)=+\infty$. So $\liminf _{y \rightarrow \infty}\left(h / h^{\prime 2}\right)=0$. Since

$$
\left.u\right|_{\partial \Omega} \leq\left.\sqrt{1-q_{0}} \sqrt{f^{2}-x^{2}}\right|_{\partial \Omega} \leq\left.\sqrt{1-q_{0}} \sqrt{h^{2}-x^{2}}\right|_{\partial \Omega},
$$

by Theorem 2.5, $u \leq \sqrt{1-q_{0}} \sqrt{h^{2}-x^{2}}=\sqrt{1-q_{0}} \sqrt{(1+b y)^{8} f^{2}-x^{2}}$ in $\Omega$; the result then follows by letting $b \rightarrow 0$.

Theorem 2.10. Let $f, \Omega$, and $u$ be as in Theorem 2.9, $\lim _{y \rightarrow \infty} f(y)=+\infty$, and $p(f) \geq p_{0}>0$, where $1 \geq p_{0}>0$ is a constant. Suppose $\left.u\right|_{\partial \Omega} \leq$ $\sqrt{1-p_{0}} \sqrt{f^{2}-x^{2}}$. Then $u \leq \sqrt{1-p_{0}} \sqrt{f^{2}-x^{2}}$ in $\Omega$.

Proof. Case 1: $0<p_{0}<1$. Let $q_{0}=\min \left(1 / 8, p_{0} / 2\right)$. Then

$$
\left.u\right|_{\partial \Omega} \leq\left.\sqrt{1-p_{0}} \sqrt{f^{2}-x^{2}}\right|_{\partial \Omega} \leq\left.\sqrt{1-q_{0}} \sqrt{f^{2}-x^{2}}\right|_{\partial \Omega} .
$$


By Theorem 2.9, $u \leq \sqrt{1-q_{0}} \sqrt{f^{2}-x^{2}}$ in $\Omega$. Let

$$
F=\sqrt{f^{2}-x^{2}} \sqrt{1-b h(y)^{-\alpha}},
$$

where $\alpha$ is some constant in $(0,1)$ to be determined later, $\varepsilon$ is a constant such that $0<\varepsilon<p_{0}$ and $b=p_{0}-\varepsilon$, and $h(y)=f\left(y_{0}+y\right)$, where $y_{0}$ is a positive constant. Since $f^{\prime}>0$ and $\lim _{y \rightarrow \infty} f=+\infty$, we can choose $y_{0}>0$ such that $h \geq 1$ for every $y>0$. Let

$$
\begin{gathered}
G=f^{2}-x^{2}, \quad g=\left(1-b h^{-\alpha}\right)^{1 / 2}, \\
g^{\prime}=\frac{1}{2}\left(1-b h^{-\alpha}\right)^{-1 / 2}(-b)(-\alpha) h^{-\alpha-1} h^{\prime} \\
=\frac{1}{2}\left(1-b h^{-\alpha}\right)^{-1 / 2} b \alpha h^{-\alpha-1} h^{\prime}, \\
g^{\prime \prime}=-\frac{1}{4}\left(1-b h^{-\alpha}\right)^{-3 / 2}(-b)(-\alpha) h^{-\alpha-1} h^{\prime} b \alpha h^{-\alpha} h^{\prime} \\
+\frac{b \alpha}{2}\left(1-b h^{-\alpha}\right)^{-1 / 2}\left((-\alpha-1) h^{-\alpha-2} h^{\prime 2}+h^{-\alpha-1} h^{\prime \prime}\right) .
\end{gathered}
$$

But $p(h) \geq p_{0}>0$, so $h^{\prime 2} \geq h h^{\prime \prime}$. Hence $g^{\prime \prime} \leq 0$. By Lemma 2.1,

$$
\begin{aligned}
G^{3 / 2} \mathfrak{M} F= & g\left(-f^{2} f^{\prime 2}+\left(f^{2}-x^{2}\right)\left(f^{\prime 2}+f f^{\prime \prime}\right)-x^{2}-\left(f^{2}-x^{2}\right)\right. \\
& \left.+\left(f^{2}-x^{2}\right) 2 f f^{\prime} \frac{g^{\prime}}{g}+G^{2} \frac{g^{\prime \prime}}{g}\right) \\
& +g^{3}\left(x^{2}\left(f^{\prime 2}+f f^{\prime \prime}\right)-f^{2} f^{\prime 2}\right. \\
& \left.\quad+x^{2}\left(G \frac{g^{\prime \prime}}{g}-3 G\left(\frac{g^{\prime}}{g}\right)^{2}\right)-\left(G_{y} G \frac{g^{\prime}}{g}+G^{2}\left(\frac{g^{\prime}}{g}\right)^{2}\right)\right) \\
\leq & g\left(-f^{2}{f^{\prime}}^{2}+\left(f^{2}-x^{2}\right)\left(f^{\prime 2}+f f^{\prime \prime}\right)\right. \\
& \left.-x^{2}-\left(f^{2}-x^{2}\right)+2 f f^{\prime} \frac{g^{\prime}}{g}\left(f^{2}-x^{2}\right)\right) \\
& +g^{3}\left(x^{2}\left(f^{\prime 2}+f f^{\prime \prime}\right)-f^{2} f^{\prime 2}\right) \\
\leq & \left(-g+g^{3}\right)(2-p(f)) x^{2} f^{\prime 2}+f^{2} f^{\prime 2} g\left((1-p(f))-g^{2}\right) \\
& -g f^{2}+2 f f^{\prime} g^{\prime}\left(f^{2}-x^{2}\right) \\
\leq & -\left(g-g^{3}\right)(2-p(f)) x^{2} f^{\prime 2}-f^{2} f^{\prime 2} g \max (p(f)-2,0) \\
& +f^{2} f^{\prime 2} g\left(\left(1-\min (2, p(f))-g^{2}\right)-g f^{2}+2 f f^{\prime} g^{\prime}\left(f^{2}-x^{2}\right) .\right.
\end{aligned}
$$

Since $1 \geq g \geq \sqrt{1-b}$,

$$
\begin{aligned}
G^{3 / 2} \mathfrak{M} F & \leq f^{2} f^{\prime 2} g\left(1-\min (2, p(f))-g^{2}\right)+2 f f^{\prime} g^{\prime} f^{2} \\
& \leq f^{2} f^{\prime 2} g\left(-\min (2, p(f))+b h^{-\alpha}\right)+2 f^{3} f^{\prime} \frac{1}{2}\left(1-b h^{-\alpha}\right)^{-1 / 2} b \alpha h^{-\alpha-1} h^{\prime} \\
& \leq f^{2} f^{\prime 2} \sqrt{1-b}\left(-\min (2, p(f))+b h^{-\alpha}\right)+b \alpha h^{-\alpha-1} h^{\prime} \frac{1}{\sqrt{1-b}} f^{3} f^{\prime} \\
& \leq f^{2} f^{\prime 2} \sqrt{1-b}(-\min (2, p(f))+b) h^{-\alpha}+b \alpha h^{-\alpha-1} h^{\prime} \frac{1}{\sqrt{1-b}} f^{3} f^{\prime} \\
& \leq h^{-\alpha} f^{2} f^{\prime 2}\left(-\sqrt{1-b} \varepsilon+\alpha b h^{-1} h^{\prime} \frac{1}{\sqrt{1-b}} \frac{f}{f^{\prime}}\right) .
\end{aligned}
$$


Since $\left(f / f^{\prime}\right)^{\prime}=p(f)>0, f / f^{\prime}$ is monotone increasing. But $h(y)=$ $f\left(y_{0}+y\right), y_{0}$ is a positive constant; therefore $\frac{h^{\prime}}{h} \frac{f}{f^{\prime}} \leq 1$. So when $0<\alpha<$ $(1-b) \varepsilon / b$, we have $G^{3 / 2} \mathfrak{M} F \leq 0$. Since

$$
\lim _{y \rightarrow \infty} f(y)=+\infty, \quad \lim _{y \rightarrow \infty} \sqrt{1-b h(y)^{-\alpha}} \rightarrow 1,
$$

we have $F(x, y) \geq \sqrt{1-q_{0}} \sqrt{f^{2}-x^{2}}$ for sufficiently large $y$. Since $u \leq$ $\sqrt{1-q_{0}} \sqrt{f^{2}-x^{2}}$ in $\Omega$ and $\left.u\right|_{\partial \Omega} \leq\left. F\right|_{\partial \Omega}$ by hypothesis, we have $u \leq F$ in $\Omega$. So $u \leq \sqrt{1-b h^{-\alpha}} \sqrt{f^{2}-x^{2}}$ in $\Omega$. Letting $\alpha \rightarrow 0, u \leq \sqrt{1-b} \sqrt{f^{2}-x^{2}}=$ $\sqrt{1-\left(p_{0}-\varepsilon\right)} \sqrt{f^{2}-x^{2}}$ in $\Omega$. Letting $\varepsilon \rightarrow 0$, we get $u \leq \sqrt{1-p_{0}} \sqrt{f^{2}-x^{2}}$ in $\Omega$.

Case 2: $p_{0}=1$. Then for any constant $p_{1}, 0<p_{1}<1$, we have $p(f) \geq 1 \geq$ $p_{1}$. Since $\left.u\right|_{\partial \Omega} \leq \sqrt{1-1} \sqrt{f^{2}-x^{2}} \leq \sqrt{1-p_{1}} \sqrt{f^{2}-x^{2}}$, by Case 1 , we have $u \leq \sqrt{1-p_{1}} \sqrt{f^{2}-x^{2}}$ in $\Omega$. Letting $p_{1} \rightarrow 1$, the result follows.

Since the case $\lim f<+\infty$ is not very important, we omit that case.

The case for a negative constant $p_{0}$ is studied in the following theorem.

Theorem 2.11. Let $f \in C^{0}[0, \infty) \cap C^{2}(0, \infty), f \geq 0, f^{\prime}>0, f_{1} \in C^{0}[0, \infty)$, $f_{1}>0, \Omega \subset\left\{(x, y) \mid-f_{1}(y)<x<f_{1}(y), y>0\right\}, u \in C^{2}(\Omega) \cap C^{0}(\bar{\Omega}), \operatorname{div} T u \geq$ 0 in $\Omega$, and $p(f) \geq p_{0}$, where $p_{0}$ is a negative constant. Then if $f^{2} \geq$ $\left(a^{2}-1\right)\left(2-p_{0}\right) f_{1}^{2} /\left(a^{2}-\left(1-p_{0}\right)\right)$ and $\left.u\right|_{\partial \Omega} \leq\left. a \sqrt{f^{2}-x^{2}}\right|_{\partial \Omega}$, where $a$ is $a$ positive constant satisfying $a^{2}-1+p_{0}>0$, we have $u \leq a \sqrt{f^{2}-x^{2}}$ in $\Omega$.

Proof. Let $f_{2}=e^{\alpha y} f$, where $\alpha$ is a positive constant. Then $f_{2}^{2} \geq f^{2} \geq$ $\left(a^{2}-1\right)\left(2-p_{0}\right) f_{1}^{2} /\left(a^{2}-\left(1-p_{0}\right)\right)$. By Lemma $2.6, p\left(f_{2}\right) \geq p_{0}$. It is easy to see that $\lim \sup _{y \rightarrow \infty}\left(f_{2} / y^{2}\right)=+\infty$, by Lemma 2.8 , and $\liminf _{y \rightarrow \infty}\left(f_{2} / f_{2}{ }^{\prime 2}\right)=0$. Since $\left.u\right|_{\partial \Omega} \leq\left. a \sqrt{f^{2}-x^{2}}\right|_{\partial \Omega} \leq\left. a \sqrt{f_{2}^{2}-x^{2}}\right|_{\partial \Omega}$, by Theorem $2.5, u \leq a \sqrt{f_{2}^{2}-x^{2}}$ $=a \sqrt{e^{2 \alpha y} f^{2}-x^{2}}$ in $\Omega$. The result then follows by letting $\alpha \rightarrow 0$.

Let $\Omega \subset\left\{-f_{1}(y)<x<f_{1}(y) \mid y>0\right\}$ and $\left.u\right|_{\partial \Omega} \leq a f_{1}$, where $a$ is a positive constant. The growing rate of $u$ is stated in the following theorem.

Theorem 2.12. Let $f_{1} \in C^{0}[0, \infty) \cap C^{2}(0, \infty), f_{1}, f_{1}^{\prime}>0, \lim f_{1}=\infty$, and $p\left(f_{1}\right) \geq p_{0} \geq 0$, where $1 \geq p_{0} \geq 0$ is a constant. Moreover, let $\Omega \subset\{(x, y) \mid$ $\left.-f_{1}(y)<x<f_{1}(y), y>0\right\}$ and $f=a f_{1}$, where $a \geq 0$ is a constant. Then if $\operatorname{div} T u \geq 0$ in $\Omega$ and $\left.u\right|_{\partial \Omega} \leq f$, we have $u \leq \sqrt{\left(a^{2}+1-p_{0}\right) f_{1}^{2}+\left(1-p_{0}\right) x^{2}}$ in $\Omega$.

Proof. Case 1: $1>p_{0} \geq 0$. Since

$$
\left.u\right|_{\partial \Omega} \leq f=a f_{1} \leq\left.\sqrt{1-p_{0}} \sqrt{\frac{a^{2} f_{1}^{2}}{1-p_{0}}+f_{1}^{2}-x^{2}}\right|_{\partial \Omega}
$$

and $p\left(\sqrt{\frac{a^{2}}{1-p_{0}}+1} f_{1}\right)=p(f) \geq p_{0}$,

$$
\begin{aligned}
u & \leq \sqrt{1-p_{0}} \sqrt{\frac{a^{2}}{1-p_{0}} f_{1}^{2}+f_{1}^{2}-x^{2}} \\
& =\sqrt{\left(a^{2}+\left(1-p_{0}\right)\right) f_{1}^{2}-\left(1-p_{0}\right) x^{2}} \text { in } \Omega
\end{aligned}
$$

by Theorem 2.10 . 
Case 2: $p_{0}=1$. For every constant $b$ where $1 \geq b \geq 0$, by Case 1 , we have $u \leq \sqrt{\left(a^{2}+(1-b)\right) f_{1}^{2}-(1-b) x^{2}}$ in $\Omega$. The result then follows by letting $b \rightarrow 0$.

Now we assume that $p\left(f_{1}\right) \geq p_{0}$ with $p_{0}$ being a negative constant, and set $f^{2}=\left(a^{2}-1\right)\left(2-p_{0}\right) f_{1}^{2} /\left(a^{2}-\left(1-p_{0}\right)\right)$, where $a>\sqrt{1-p_{0}}$ is a constant. If $\left.u\right|_{\partial \Omega} \leq\left. a \sqrt{f^{2}-x^{2}}\right|_{\partial \Omega} \leq a \sqrt{\left(a^{2}-1\right)\left(2-p_{0}\right) /\left(a^{2}-\left(1-p_{0}\right)\right)-1} f_{1}$, by Theorem 2.11 , we have $u \leq a \sqrt{f^{2}-x^{2}}$ in $\Omega$. Now we want to compute the minimum of $a \sqrt{\left(a^{2}-1\right)\left(2-p_{0}\right) /\left(a^{2}-\left(1-p_{0}\right)\right)-1}$. For convenience, let $b=a^{2}$ and $q_{1}=1-p_{0}>1$, and we need to compute the minimum of

$$
b\left(\frac{(b-1)\left(1+q_{1}\right)}{b-q_{1}}-1\right)=b \frac{q_{1} b-1}{b-q_{1}} .
$$

Take logarithms and differentiate the above function; we have $1 / b+q_{1} /\left(q_{1} b-1\right)$ $=1 /\left(b-q_{1}\right)$. From this, we get $b=q_{1} \pm \sqrt{q_{1}^{2}-1}$. Since $b=a^{2}>q_{1}$, we have $b=q_{1}+\sqrt{q_{1}^{2}-1}$. So the minimum of $b\left(q_{1} b-1\right) /\left(b-q_{1}\right)$ is

$$
\left(q_{1}+\sqrt{q_{1}^{2}-1}\right)\left(\frac{q_{1}^{2}+q_{1} \sqrt{q_{1}^{2}-1}-1}{\sqrt{q_{1}^{2}-1}}\right)=\left(q_{1}+\sqrt{q_{1}^{2}-1}\right)^{2}
$$

and the minimum of $a \sqrt{\left(a^{2}-1\right)\left(2-p_{0}\right) /\left(a^{2}-\left(1-p_{0}\right)\right)-1}$ is $q_{1}+\sqrt{q_{1}^{2}-1}$.

Theorem 2.13. Assume that $f_{1}, f_{1}^{\prime}>0, p\left(f_{1}\right) \geq p_{0}$, where $p_{0}$ is a negative constant, $\Omega \subset\left\{-f_{1}(y)<x<f_{1}(y) \mid y>0\right\}$, and $u \in C^{2}(\Omega) \cap C^{0}(\boldsymbol{\Omega})$. If $\operatorname{div} T u \geq 0$ in $\Omega, q_{1}=1-p_{0}>1$, and $\left.u\right|_{\partial \Omega} \leq b f_{1}$, where $b \geq q_{1}+\sqrt{q_{1}^{2}-1}$ is a positive constant, then $u \leq \sqrt{b} \sqrt{(b+1) f^{2}-x^{2}}$ in $\Omega$.

Proof. By the assumption that $b \geq q_{1}+\sqrt{q_{1}^{2}-1}$, we have

$$
\left(b-\left(q_{1}+\sqrt{q_{1}^{2}-1}\right)\right)\left(b-\left(q_{1}-\sqrt{q_{1}^{2}-1}\right)\right) \geq 0
$$

$b^{2}-2 q_{1} b+1 \geq 0$, and $(b+1)\left(b-q_{1}\right) \geq(b-1)\left(1+q_{1}\right)$. If we set $a=b^{2}$, then $\left(a^{2}+1\right)\left(a^{2}-q_{1}\right) \geq\left(a^{2}-1\right)\left(1+q_{1}\right)$ and we have $a^{2}+1 \geq\left(a^{2}-1\right)\left(1+q_{1}\right) /\left(a^{2}-q_{1}\right)$. Since $\left.u\right|_{\partial \Omega} \leq\left. a \sqrt{\left(a^{2}+1\right) f_{1}^{2}-x^{2}}\right|_{\partial \Omega}$, by Theorem 2.11,u $\leq a \sqrt{\left(a^{2}+1\right) f_{1}^{2}-x^{2}}$ in $\Omega$ and we have $u \leq \sqrt{b} \sqrt{(b+1) f^{2}-x^{2}}$ in $\Omega$.

By Theorems 2.12 and 2.13, we know that the growth rate of $u$ is of the same order as the shape of $\Omega$ and $\left.u\right|_{\partial \Omega}$.

\section{ACKNOWLEDGMENT}

The author would like to thank the referee for many helpful comments and suggestions. 


\section{REFERENCES}

1. R. Finn, Equilibrium capillary surfaces, Springer-Verlag, New York, Berlin, Heidelberg, and Toykyo, 1986.

2. D. Gilbarg and N. S. Trudinger, Elliptic partial differential equations of second order, second ed., Springer-Verlag, Berlin, Heidelberg, New York, and Tokyo, 1983.

3. J. Hwang, Phragmén-Lindelöf theorem for the minimal surface equation, Proc. Amer. Math. Soc. 104 (1988), 825-828.

4. J. C. C. Nitsche, On new results in the theory of minimal surfaces, Bull. Amer. Math. Soc. 71 (1965), 195-270.

5. —_, Vorlesungen über Minimalflächen, Springer-Verlag, Berlin, Heidelberg, and New York, 1975.

6. R. Osserman, A survey of minimal surfaces, Van Nostrand-Reinhold, New York, 1969.

7. M. H. Protter and H. F. Weinberger, Maximum principles in differential equations, Prentice-Hall, Englewood Cliffs, NJ, 1967.

Institute of Mathematics, Academia Sinica, Taipei 11529, Taiwan, Republic of China 